\title{
Bottom meson masses from a RHQ action on a fine 2+1 flavor DWF lattice
}

\author{
Hao Peng* \\ Department of Physics, Columbia University \\ E-mail: hp2151@columbia.edu
}

\begin{abstract}
We extend our previous relativistic heavy quark (RHQ) charm calculations performed on the $32^{3} \times 64,1 / a=2.32 \mathrm{GeV} \mathrm{RBC} / \mathrm{UKQCD}$ lattice ensembles to states including bottom quarks. We calculate masses of bottomium and bottom-strange states, match and compare them to known experimental values. We also compare our results with those determined on the $24^{3} \times 64,1 / a=$ $1.73 \mathrm{GeV}$ to assess the RHQ method.
\end{abstract}

The XXVIII International Symposium on Lattice Field Theory, Latt ice2010

June 14-19, 2010

Villasimius, Italy

\footnotetext{
* Speaker.
} 


\section{Introduction}

The study of mesons containing heavy quarks plays an important role in particle physics by helping constrain the standard model parameters. The first-principle methods of lattice quantum chromodynamics (QCD) have been used to obtain some of the most accurate theoretical information so far, and connect to the experimental observations of those hadronic properties. However, the relatively large masses of charm and bottom quarks $\left(m_{c}=1.25 \mathrm{GeV}, m_{b}=4.20 \mathrm{GeV}\right)$ make it impractical to work with lattice spacing sufficiently small to properly control discretization errors of $O(m a)$ [1]. To overcome this problem, we have adopted the Fermilab or the relativistic heavy quark (RHQ) method[2, 3, 4], which takes the following form:

$$
S_{l a t}=\sum \bar{\psi}\left(m_{0} a+\gamma_{0} D_{0}+\zeta \vec{\gamma} \cdot \vec{D}-\frac{1}{2}\left(D^{0}\right)^{2}-\frac{1}{2} \zeta(\vec{D})^{2}+\sum_{\mu, v} \frac{i}{4} c_{p} \sigma_{\mu v} F_{\mu \nu}\right) \psi
$$

It only contains three parameters to tune: the Wilson quark mass $m_{0} a$, an asymmetry parameter $\zeta$ describing the ratio of the coefficients of the spatial and temporal derivative, and $c_{p}$, a generalization of the Sheikholeslami and Wohlert term to the case of nonzero mass. By adjusting these coefficients properly, this action can accurately describe heavy quark systems. The hardronic masses computed in the resulting theory will contain errors no larger than $(\vec{p} a)^{2}[2,3,4]$.

The three RHQ parameters can be obtained by matching to the experimental data, if lattice spacing is known. In earlier studies, this has been successfully done on the RBC/UKQCD $24^{3} \times 64$ lattice ensembles[5], and on the $32^{3} \times 64$ ensembles for the charm system[6].

\section{Methods}

We have calculated bottomium and bottom-strange correlators using the RHQ action of eq. 1.1 , on a 1k-node partition of the QCDOC machines in the RIKEN BNL Research Center (RBRC). The pseudoscalar, vector, scalar, and axial-vector state meson masses are determined from the time dependence of the correlators. Following our old convention in Ref.[7], we work with the spinaveraged mass, $\left(m_{B_{s}}+3 m_{B_{s}^{*}}\right) / 4$, the hyperfine splitting, $\left(m_{B_{s}^{*}}-m_{B_{s}}\right)$, and the mass ratio, $m_{1} / m_{2}$, obtained from the dispersion relation, $E^{2}=m_{1}^{2}+\frac{m_{1}}{m_{2}} p^{2}$, with $m_{1}$ the rest mass and $m_{2}$ the kinetic mass. By matching these two meson masses/splittings to the experiment results and requiring $m_{1} / m_{2}=1$, we can then determine the three parameters $m_{0} a$, $\zeta$ and $c_{p}$.

To do the matching, we choose a sufficiently small region in the parameter space, so that meson masses are well approximated by a simple linear dependence on the heavy quark parameters:

$$
Y=A+J \cdot X
$$

where $X$ is a vector of input RHQ parameters, $X^{T}=\left(m_{0} a, c_{p}, \zeta\right)$, and $Y$ is made up of computed masses and mass ratios, e.g., $Y^{T}=\left(m_{s a} a, m_{h s} a, m_{1} / m_{2}\right)$. The quantities $A$ and $J$ represent the linear coefficients. In our calculation we start with the seven sets of initial RHQ parameters shown in Figure 1. Then $A$ and $J$ can be simply determined by taking the derivative of each component of $Y$ in each direction through differencing. In the end the RHQ parameters $X_{c}$ are determined explicitly as

$$
X_{c}=J^{-1} \cdot\left(Y_{e x p}-A\right)
$$


where $Y_{\text {exp }}$ is from PDG. The error on $X_{c}$ is calculated from the jackknifed results of $J$ and $A$ which represent the statistical fluctuations in our lattice ensembles. With known RHQ parameters, we can make predictions in the reversed way:

$$
Y_{P}=A_{P}+J_{P} \cdot X_{c}
$$

where the vector $Y_{P}$ consists of predicted meson masses, e.g., $m_{\eta_{b}}, m_{\Upsilon}, m_{\chi_{b 0}}, m_{\chi_{b 1}} ; J_{P}$ and $A_{P}$ are linear coefficients of these states calculated in the same way as above.
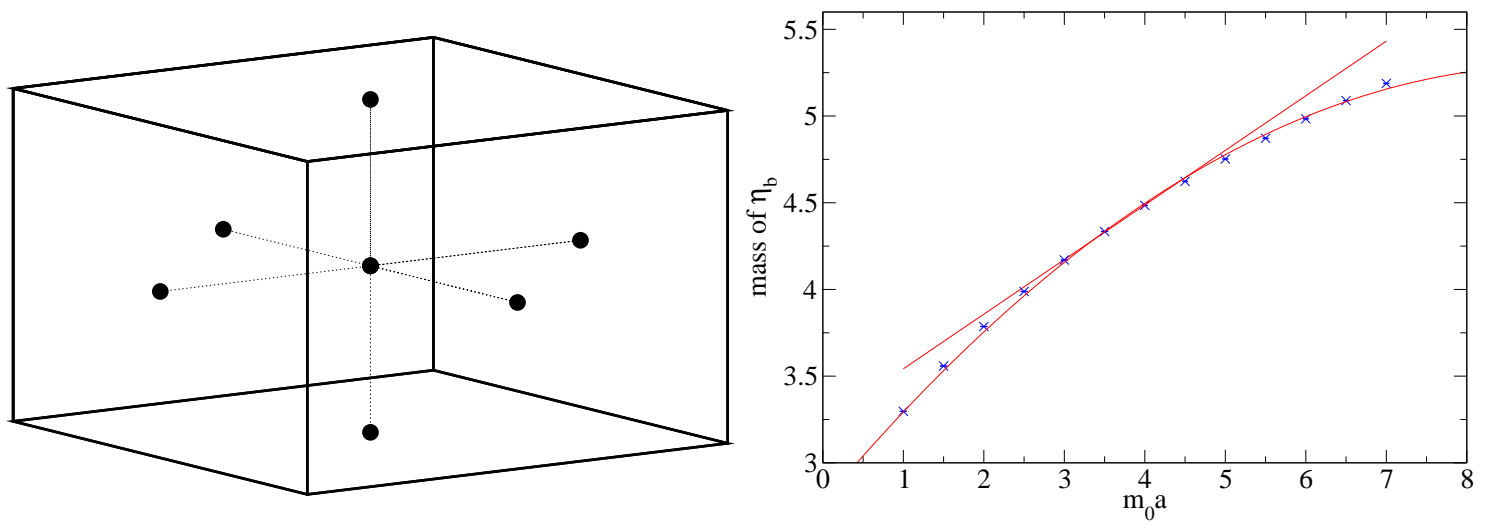

Figure 1: The 7 initial trial RHQ parameters are $\left(m_{0} a=3.4 \pm 0.5, c_{p}=3 \pm 1, \zeta=2.5 \pm 0.3\right)$, distributed as shown in the box(left). It seems bottom meson masses show strong nonlinear dependence on the RHQ parameters in the long range(right). So we require the box size to be small enough, and determined RHQ parameters must be inside the box.

\section{Preliminary Results}

We calculate correlators by combining box-source bottom propagators and wall-source strange propagators produced by the RBC-UKQCD collaboration on $32^{3} \times 64$ lattices, the details of which are shown in Table 1. Sample effective mass plots are shown in Figure 2, and the meson masses are obtained by doing a hyperbolic cosine fit over a carefully chosen plateau. The mass ratio is evaluated from the dispersion relation of one meson, by linear regression, shown in Figure 3. Throughout our calculation, we use a lattice spacing of $a^{-1}=2.32 \mathrm{GeV}$, from Ref.[8].

\begin{tabular}{ccccc}
\hline \hline volume & $L_{s}$ & $\left(m_{\text {sea }}, m_{s}\right)$ & Trajectory(step) & \# of configs \\
\hline $32^{3} \times 64$ & 16 & $(0.004,0.025)$ & $260-3320(10)$ & 289 \\
$32^{3} \times 64$ & 16 & $(0.006,0.025)$ & $530-3760(10)$ & 213 \\
$32^{3} \times 64$ & 16 & $(0.008,0.025)$ & $260-2880(10)$ & 254 \\
\hline \hline
\end{tabular}

Table 1: The dynamical 2+1 flavor $\beta=2.25$ lattice configurations used for our bottomium and bottomstrange calculators. The molecular dynamics time interval between two sequential measurements is 20 . A single source is placed at $0,16,32$ or 48 to minimize inter-correlations. The size of the box-source is set to be 8 . The fitting range for $B_{s}, B_{s}^{*}$ is 11-25, for $\eta_{b}, \Upsilon$ is $17-30$ and for $\chi_{b 0}, \chi_{b 1}$ is $9-18$.

In the charm system we determined the RHQ parameters competely from the charmonion correlators. However, in the bottom system, we do not match with bottomium meson masses 

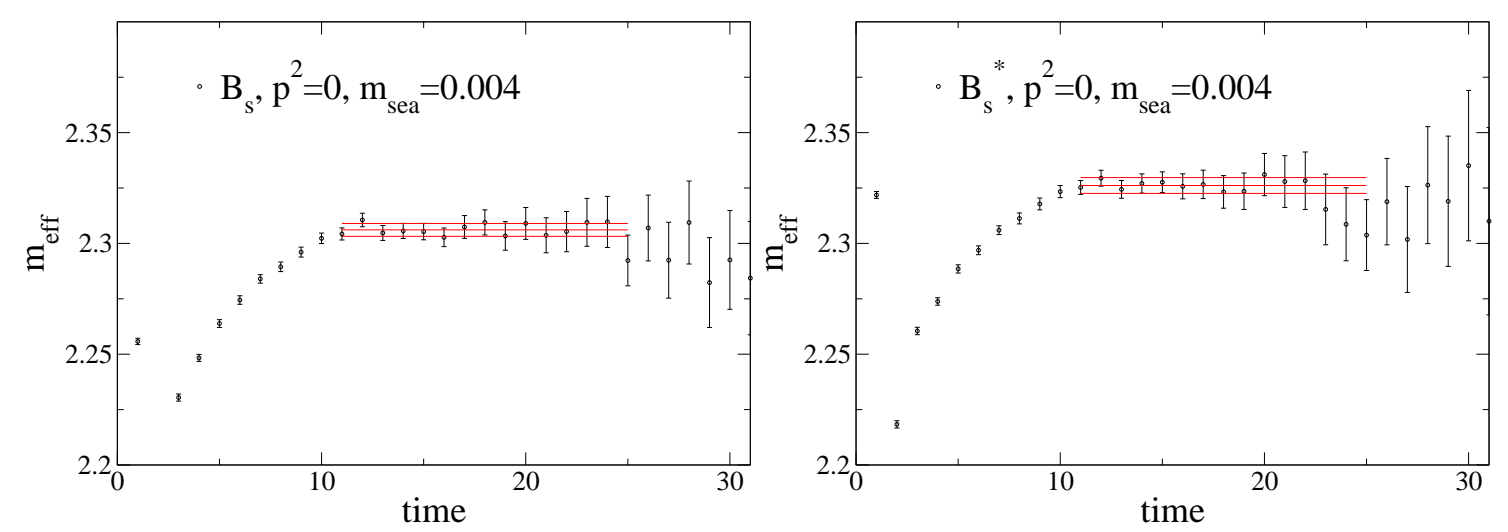

Figure 2: Sample effective mass plots of pseudo-scalar and vector states from bottom-strange correlators. The red lines are the fitted results with one standard deviation error bands.

$m_{\eta_{b}}$ or $m_{\Upsilon}$, since this approach could involve a large systematic error, estimated as $O\left(p^{2} a^{2}\right) \sim$ $\left(\alpha_{s} m_{b}\right)^{2} \sim\left(1 \mathrm{GeV} \cdot a_{32}\right)^{2} \sim 20 \%$. Instead we choose $m_{B_{s}}, m_{B_{s}^{*}}$ to minimize the systematic error, which is $O\left(p^{2} a^{2}\right) \sim\left(\Lambda_{Q C D}\right)^{2} \sim\left(300 \mathrm{MeV} \cdot a_{32}\right)^{2} \sim 2 \%$, but we will introduce a larger statistical error in this way. A third approach was taken in the calculations on $24^{3} \times 64$ lattices[5], where $m_{1} / m_{2}$ was determined from $\eta_{b}$ or $\Upsilon$, while $m_{B_{s}}$ or $m_{B_{s}^{*}}$ provided the other two inputs. A reduced statistical error but an increased systematic error will result from this hybrid approach.
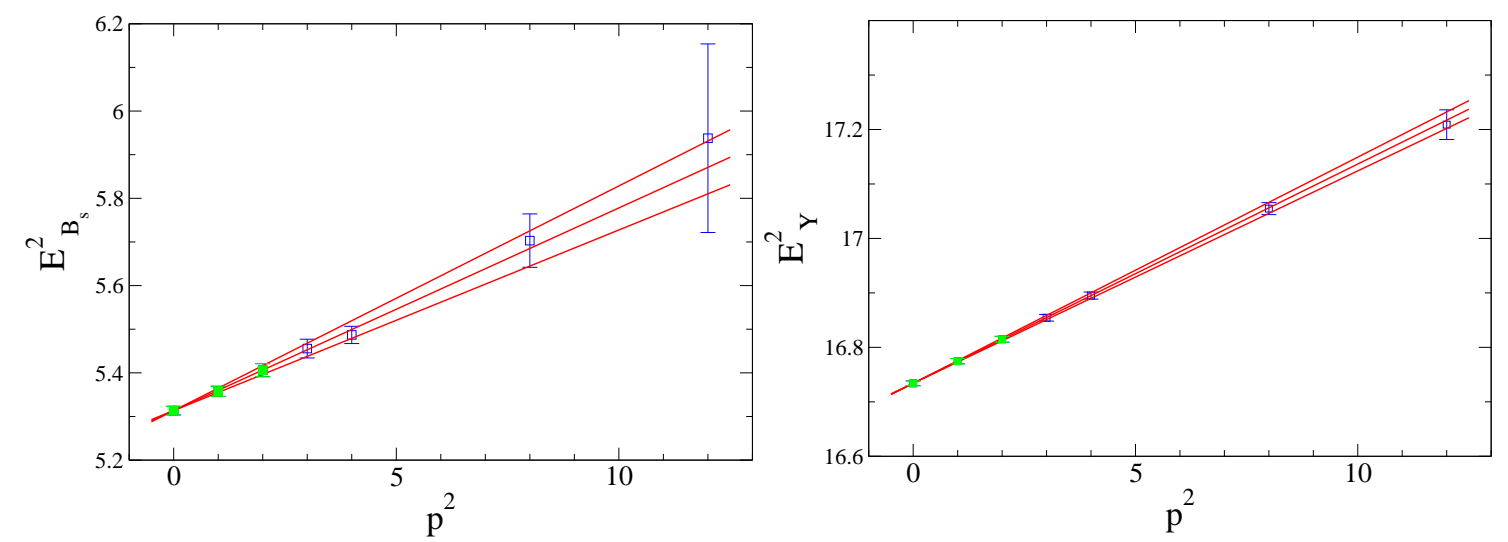

Figure 3: Dispersion relation of $B_{s}$ and $\Upsilon$ on the ensemble of $m_{\text {sea }}=0.004$. Fits are based on the first 3 momenta(green points), i.e., $p^{2}=0,1,2$. The greater precisions of $E_{\Upsilon}$ can be easily seen.

\subsection{RHQ parameters and predictions}

The resulting RHQ parameters and corresponding predicted meson masses are listed in Table 2 and 3. The values of the RHQ parameters and the predicted masses at $m_{\text {phys }}=0.000399$ are obtained by linear extrapolation from those on the three ensembles, as shown in Figure 4 and 5. We find the RHQ parameters listed in the three seperate sections of Table 2 at $m_{\text {phys }}$ agree with each other within one standard deviation. The results using $\left(m_{1} / m_{2}\right) \Upsilon$ have a small statistical error, but could include a large systematic bias. On the other hand, using $\left(m_{1} / m_{2}\right)_{B_{s}}$ or $\left(m_{1} / m_{2}\right)_{B_{s}^{*}}$ gives a much larger statistical errors, because the input value $m_{1} / m_{2}$ has larger errors than that from the bottomium system. To avoid large systematic errors, which are difficult to estimate reliably, we 


\begin{tabular}{c|c|ccc}
\hline \hline$m_{\text {sea }}$ & Inputs & $m_{0} a$ & $c_{p}$ & $\zeta$ \\
\hline 0.004 & $m_{B_{s}}, m_{B_{s}^{*}},\left(m_{1} / m_{2}\right) \Upsilon$ & $3.57(11)$ & $3.36(45)$ & $2.42(6)$ \\
0.006 & $m_{B_{s}}, m_{B_{s}^{*}},\left(m_{1} / m_{2}\right)_{\Upsilon}$ & $3.49(12)$ & $2.98(49)$ & $2.41(5)$ \\
0.008 & $m_{B_{s}}, m_{B_{s}^{*}},\left(m_{1} / m_{2}\right) \Upsilon$ & $3.56(12)$ & $3.37(52)$ & $2.42(5)$ \\
chiral & $m_{B_{s}}, m_{B_{s}^{*}},\left(m_{1} / m_{2}\right) \Upsilon$ & $3.56(23)$ & $3.28(97)$ & $2.41(12)$ \\
\hline 0.004 & $m_{B_{s}}, m_{B_{s}^{*}},\left(m_{1} / m_{2}\right)_{B_{s}}$ & $3.72(26)$ & $3.61(66)$ & $2.24(30)$ \\
0.006 & $m_{B_{s}}, m_{B_{s}^{*}},\left(m_{1} / m_{2}\right)_{B_{s}}$ & $3.32(25)$ & $2.79(56)$ & $2.67(35)$ \\
0.008 & $m_{B_{s}}, m_{B_{s}^{*}},\left(m_{1} / m_{2}\right)_{B_{s}}$ & $4.30(69)$ & $4.83(1.52)$ & $1.59(84)$ \\
chiral & $m_{B_{s}}, m_{B_{s}^{*}},\left(m_{1} / m_{2}\right)_{B_{s}}$ & $3.72(72)$ & $3.41(1.74)$ & $2.28(85)$ \\
\hline 0.004 & $m_{B_{s}}, m_{B_{s}^{*}},\left(m_{1} / m_{2}\right)_{B_{s}^{*}}$ & $3.67(31)$ & $3.53(71)$ & $2.31(36)$ \\
0.006 & $m_{B_{s}}, m_{B_{s}^{*}},\left(m_{1} / m_{2}\right)_{B_{s}^{*}}$ & $3.12(47)$ & $2.61(84)$ & $2.97(63)$ \\
0.008 & $m_{B_{s}}, m_{B_{s}^{*}},\left(m_{1} / m_{2}\right)_{B_{s}^{*}}$ & $4.38(1.07)$ & $4.99(2.59)$ & $1.50(1.08)$ \\
chiral & $m_{B_{s}}, m_{B_{s}^{*}},\left(m_{1} / m_{2}\right)_{B_{s}^{*}}$ & $3.75(97)$ & $3.88(2.16)$ & $2.44(1.09)$ \\
\hline \hline
\end{tabular}

Table 2: The RHQ parameters determined from different approaches.

\begin{tabular}{c|ccc|c|c}
\hline \hline$m_{\text {sea }}$ & 0.004 & 0.006 & 0.008 & chiral & experiment \\
\hline$m_{\chi_{b 0}}$ & $9874(52)$ & $9944(57)$ & $9777(135)$ & $98883(144)$ & $9859(3)$ \\
$m_{\chi_{b 1}}$ & $9910(49)$ & $9967(57)$ & $9827(127)$ & $9922(136)$ & 9893 \\
$m_{\chi_{b 1}}-m_{\chi_{b 0}}$ & $36.3(7.7)$ & $23.6(6.4)$ & $49.4(17.0)$ & $35.6(20.1)$ & 33.34 \\
\hline$m_{\eta_{b}}$ & $9452(42)$ & $9502(51)$ & $9355(113)$ & $9474(119)$ & $9389(3)(3)$ \\
$m_{\Upsilon}$ & $9499(39)$ & $9537(50)$ & $9420(102)$ & $9524(110)$ & 9460 \\
$m_{\Upsilon}-m_{\eta_{b}}$ & $46.4(9.6)$ & $34.7(8.1)$ & $65.1(22.3)$ & $43.0(25.5)$ & $71(3)(3)$ \\
\hline
\end{tabular}

Table 3: Predicted Bottom meson masses. All quantities are in unit of $\mathrm{MeV}$

will calculate everything using $m_{1} / m_{2}$ from the heavy-strange system. These large statistical errors can be reduced by adding more lattice configurations. Another problem we notice is that for the $m_{\text {sea }}=0.008$ ensemble, the linearly determined RHQ parameters fall outside the box formed by our seven parameter choices as shown in Figure 1, which applifies their statistical errors relative to the other two ensembles because of the linear extrapolation instead of interpolation. Again more statistics are required.

The predicted meson masses are based on the RHQ parameters in the 2nd section of Table 2, using $\left(m_{1} / m_{2}\right)_{B_{s}}$, which have smaller errors compared to $\left(m_{1} / m_{2}\right)_{B_{s}^{*}}$. It turns out our $m_{\chi_{b 0}}$, $m_{\chi_{b 1}}, m_{e t a_{b}}$ and $m_{\Upsilon}$ agree with the experimental results within one standard deviation. However, the large statistical errors, which are above $100 \mathrm{MeV}$, limit the accuracy of this test of the RHQ prediction method. The hyper-fine mass splitting, $\left(m_{\Upsilon}-m_{\eta_{b}}\right)$, is about half of the experimental value, similar to what was found on the $24^{3} \times 64$ lattices[5], which may indicate a larger systematic errors expected when the RHQ method is applied to a system of two heavy quarks.

\subsection{Scaling Limit}

We present some predicted bottom meson masses from our past lattice calculations in Figure 6. The masses from the $24^{3} \times 64$ lattices are re-calculated using $\left(m_{1} / m_{2}\right)_{B_{S}^{*}}$ instead of originally 

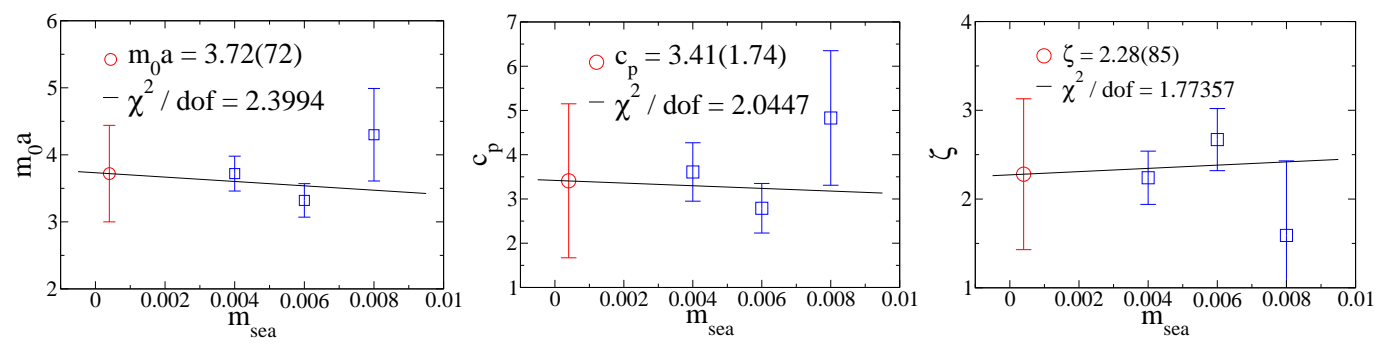

Figure 4: The RHQ parameters extrapolated to the physical light quark mass limit at $m_{p h y s}=0.000399$.
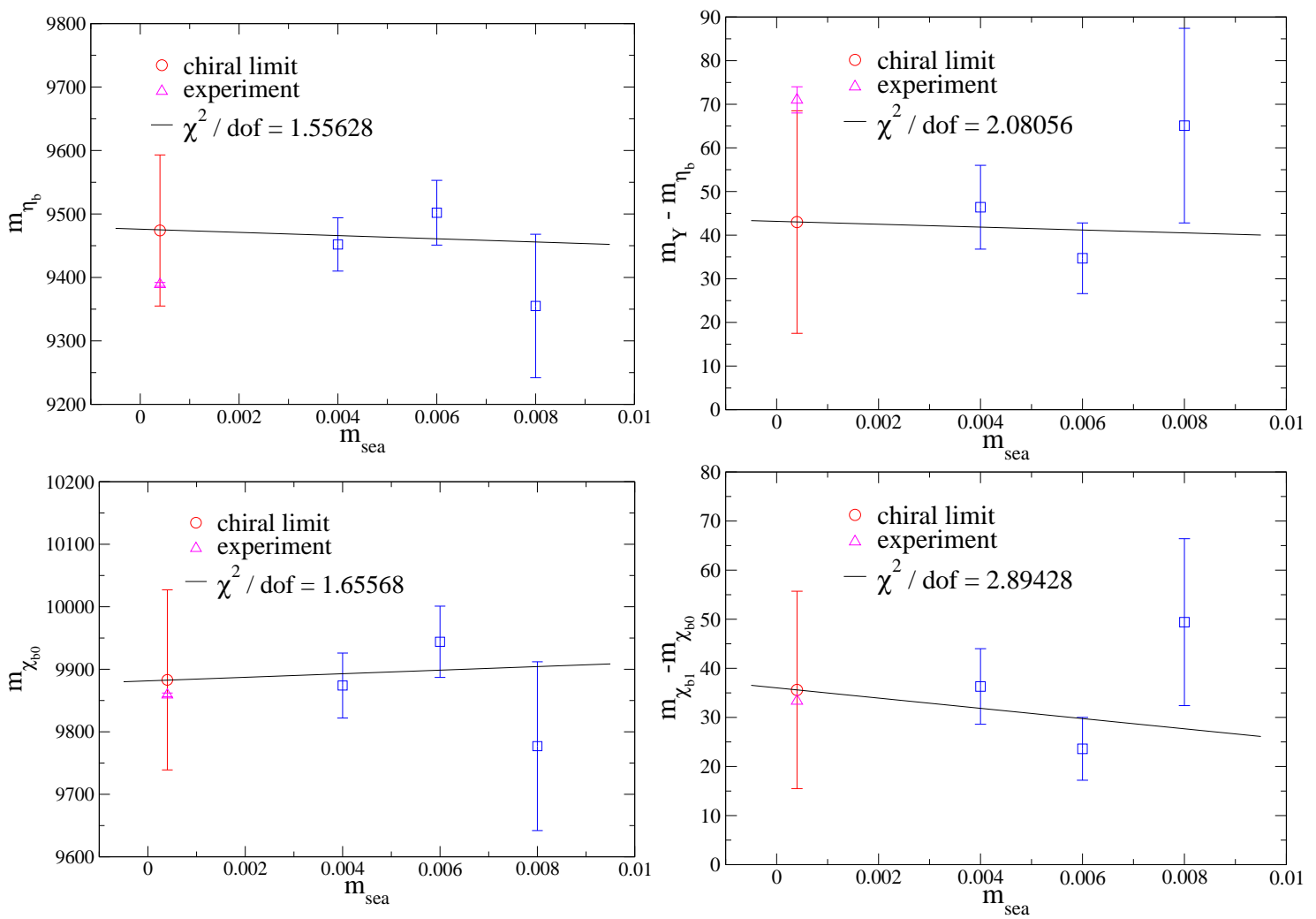

Figure 5: The predicted bottom meson masses extrapolated to the physical light quark mass limit, $m_{\text {phys }}=0.000399$

chosen $\left(m_{1} / m_{2}\right)_{r}$ [5], so that we can make a comparison based entirely on heavy-strange correlators. Unfortunately, the present errors are too large to place more than a crude bound on the $a^{2}$ dependence of the bottom masses.

\section{Conclusions}

In this proceedings we have illustrated the method of tuning the RHQ parameters using $32^{3} \times$ 64 lattices, and data entirely from heavy-strange systems. We find, however, that the statistical errors in the tuned RHQ parameters are quite large, and we plan to reduce them by adding statistics. We emphasize that these results are preliminary, and we expect them to change before a final publication. The final tuned RHQ parameters will be used to compute the $B_{d}$ and $B_{s}$ meson decay 

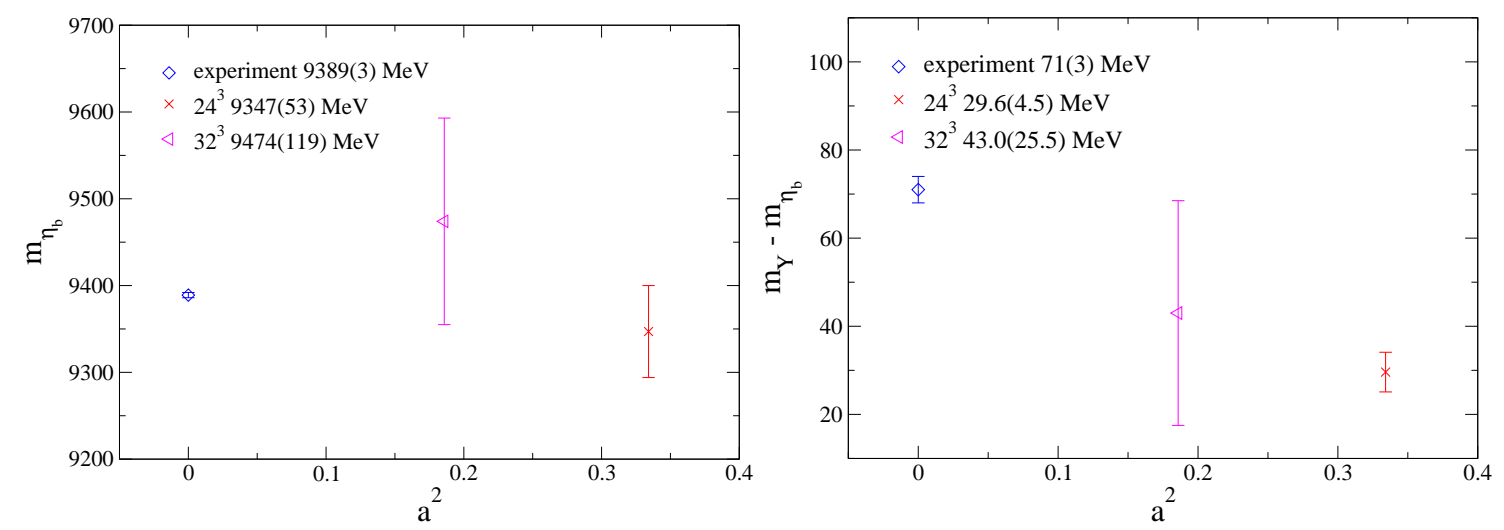

Figure 6: The masses of $\chi_{b 0}$ and $\chi_{b 1}$ at different lattice spacings.

constants and mixing parameters[9], which are important quantities for flavor physics phenomenology.

\section{Acknowledgements}

Our work was supported by DOE Grant No.DE-FG02-92ER40699. I would like to thank Norman Christ, Oliver Witzel, Ruth Van de Water, Yasumichi Aoki, Taku Izubuchi, Xiao-Yong Jin, Chulwoo Jung, Matthew Lightman, Qi Liu, Robert Mawhinney and all other members in the RBC collaboration for their help through the work. I also thank the RBRC and the the BNL for providing facilities essential to this work.

\section{References}

[1] A. S. Kronfeld, Heavy quarks and lattice QCD, Nucl. Phys. Proc. Suppl. 129 (2004) 46-59, [hep-lat/0310063].

[2] A. X. El-Khadra, A. S. Kronfeld, and P. B. Mackenzie, Massive Fermions in Lattice Gauge Theory, Phys. Rev. D55 (1997) 3933-3957, [hep-lat/9604004].

[3] S. Aoki, Y. Kuramashi, and S.-i. Tominaga, Relativistic heavy quarks on the lattice, Prog. Theor. Phys. 109 (2003) 383-413, [hep-lat/ 0107009 ].

[4] N. H. Christ, M. Li, and H.-W. Lin, Relativistic heavy quark effective action, Phys. Rev. D76 (2007) 074505, [hep-lat/0608006].

[5] M. Li and H.-W. Lin, Charm spectroscopy on dynamical 2+1 flavor domain wall fermion lattices with a relativistic heavy quark action, PoS LAT2007 (2007) 117, [0710 . 0910].

[6] RBC and UKQCD Collaboration, H. Peng, Toward the scaling limit of the charm spectrum using domain wall and relativistic heavy quark, PoS LAT2009 (2009) 094.

[7] H.-W. Lin and N. Christ, Non-perturbatively determined relativistic heavy quark action, Phys. Rev. D76 (2007) 074506, [hep-lat / 0608005$].$

[8] RBC and UKQCD Collaboration, R. Mawhinney, Nlo and nnlo chiral fits or 2+1 flavor $d w f$ ensembles, PoS LAT2009 (2009) 081.

[9] RBC Collaboration, R. S. Van de Water and O. Witzel, B-physics with dynamical domain-wall light quarks and relativistic b-quarks, PoS LAT2010 (2010) 318. 